\title{
Olfactory Function in Patients with Non-syndromic Orofacial Clefts and their Unaffected
}

\section{Relatives}

Jasmien Roosenboom ${ }^{1}$, Robert Hermans ${ }^{2}$, Frederik Lammens ${ }^{3}$, Jean Louis Samain ${ }^{4}$, Koen Devriendt ${ }^{5,6}$, Vincent Vander Poorten ${ }^{3,6}$, Peter W. Hellings ${ }^{3,6}$, Mark Jorissen ${ }^{3}$, Hilde Peeters ${ }^{5}$, Peter Claes ${ }^{7}$, Greet Hens ${ }^{3,6}$

${ }^{1}$ Department of Neurosciences, Research Group Experimental Otorhinolaryngology, Catholic University Leuven, Leuven, Belgium

${ }^{2}$ Radiology Department, University Hospitals Leuven, Leuven, Belgium

${ }^{3}$ Department of Otorhinolaryngology, Head and Neck Surgery, University Hospitals Leuven, Leuven, Belgium

${ }^{4}$ Radiology Department, Centre Hospitalier de Mouscron, Mouscron, Belgium

${ }^{5}$ Center for Human Genetics, University Hospitals Leuven, Leuven, Belgium

${ }^{6}$ Multidisciplinary Cleft Team, University Hospitals Leuven, Leuven, Belgium

${ }^{7}$ Department of Electrical Engineering, Medical Image Computing, ESAT/PSI, Catholic University Leuven, Leuven, Belgium

Running title: Olfactory Dysfunction in Orofacial Clefting

Corresponding author:

Prof. Dr. Greet Hens

Kapucijnenvoer 7 blok h - bus 7001

3000 Leuven

Belgium

Greet.hens@uzleuven.be 
Roosenboom et al. 2 Olfactory Dysfunction in Orofacial Clefting

Tel: +3216 372911

Fax: +3216 332355 


\section{Abstract}

Non-syndromic orofacial clefting is one of the most frequently occuring congenital conditions. The aim of the study was to investigate the prevalence and nature of reduced olfactory function in patients with non-syndromic cleft lip and/or cleft palate (NSCL/P) and their unaffected firstdegree relatives. Olfactory function was tested using the Sniffin' Sticks identification test in patients with NSCL/P, in their unaffected relatives, and in control subjects. MR imaging was performed to measure olfactory bulb (OB) volumes and olfactory sulcus (OS) depths. A reduced olfactory function was seen in significantly more patients with NSCL/P $(p=0,002)$ than in control subjects, regardless of the cleft type. Strikingly, unaffected relatives of patients with NSCL/P also had a higher rate of hyposmia $(\mathrm{p}=0,001)$. In hyposmic patients, the OB volumes (left: $p=0,01$ and right: $p=0,003)$ and the depth of the left $O S(p=0,02)$ were significantly smaller than in controls. In hyposmic relatives, both OS depths (left: $\mathrm{p}=0,02$ and right: $\mathrm{p}=0,03$ ) were significantly smaller. Patients with NSCL/P and their unaffected relatives have an increased prevalence of reduced olfactory function, associated with changes in the central olfactory structures.

Key words: Cleft lip and palate, Smell, Endophenotype, Olfactory bulb, Olfactory sulcus 


\section{INTRODUCTION}

Non-syndromic cleft lip with/without cleft palate (NSCL/P) is one of the most frequently occurring congenital conditions with an incidence of 1 in 700 live births (Stanier, 2004). Orofacial clefts are responsible for a major social and psychological burden in the lives of the patients and their families and require a long and multidisciplinary follow-up until adulthood (Marazita, 2012). The etiology of NSCL/P is considered to be multifactorial, but both the genetic and environmental contributing factors remain incompletely understood.

Endophenotypes are characteristics (behavioral or anatomical) associated with a condition, which are also present in unaffected family members (Leboyer et al., 1998). They might be an expression of underlying susceptibility genes for the condition. An endophenotype is by definition a characteristic that is associated with a condition, is heritable, can manifest in an individual independently of the condition, co-segregates within a family and has a higher prevalence in unaffected family members compared to the general population (Gottesman \& Gould, 2003). Several endophenotypes are described in unaffected first-degree relatives of patients with NSCL/P, such as specific facial characteristics, a deviation of the anatomy of the orbicularis oris muscle and whorl patterns on the lower lip (Roosenboom et al., 2015).

In this study, we hypothesize that reduced olfactory function is another endophenotype in NSCL/P. Although the olfactory function of patients with NSCL/P has not been extensively investigated, there is some evidence for a decreased capacity to smell in patients with NSCL/P. Richman et al. described reduced smell identification in boys with isolated cleft palate (CP), compared with healthy controls (Richman et al., 1988). This was not the case in girls with CP. Grossmann et al. showed a higher smell threshold in patients with CP, without impairment of the smell identification (Grossmann et al., 2005). Mani et al. found significant reduced smell identification in patients with unilateral cleft lip with/without cleft palate (CL/P) (Mani et al., 
2010). May et al. found a higher prevalence of reduced olfactory function in parents of patients with NSCL/P compared with normative data (May, Sanchez, Deleyiannis, Marazita, \& Weinberg, 2015). Recently, we were able to describe reduced olfactory function in unaffected first-degree relatives of patients with NSCL/P and link this endophenotype to a reduced upper nasal area (Roosenboom et al., 2015). This supports the idea that reduced olfactory function in patients with NSCL/P and their unaffected relatives may be an expression of variants in certain susceptibility factors for CL/P. In that case, reduced olfactory function can be considered as an endophenotype of CL/P.

We investigated olfactory function in patients with NSCL/P and their unaffected firstdegree relatives by standardized olfactory testing as compared to a control group without family history of orofacial clefting. In addition, MRI assessment of olfactory bulb (OB) volumes and olfactory sulcus (OS) depths was performed.

\section{MATERIALS AND METHODS}

\section{Subjects}

Fifty-four patients with NSCL/P (27 males and 27 females, mean age of 29,4 \pm 10.39 years) and 44 unaffected first-degree relatives of patients with NSCL/P (17 males and 27 females, mean age of $44,3 \pm 8.75$ years) were recruited at the Multidisciplinary Cleft Clinic of the University Hospitals Leuven, Belgium. Simultaneously, a control group of 35 persons with a negative familial history for NSCL/P was recruited at the outpatient ENT clinic of the University Hospitals Leuven, Belgium (11 males and 24 females, mean age of 40,0 \pm 13.66 years) (Table 1). 12 of the 44 relatives could be obligate carriers for susceptibility genes for NSCL/P (i.e. unaffected relatives who, according to their pedigree, are the link between two affected individuals in different generations and are thus, under the hypothesis of a multifactorial model, potential carriers of susceptibility genes). Two of these obligated carriers 
were related to each other (one parent and one sibling of the same subject). In the patient group, 18 patients had an isolated CP, 7 had an isolated cleft lip (CL) and 29 had a cleft lip and palate (CLP). Exclusion criteria for all participants were an age under 16 years, intake of medication that could lead to a reduction in olfactory function and a history of inflammatory rhinological disease or of significant nasal obstruction due to structural anomalies. The subjects were questioned about their medical history, with relation to nasal obstruction and rhinological disease. Significant structural or inflammatory nasal anomalies were excluded by clinical examination and by anterior rhinoscopy, acoustic rhinometry and rhinomanometry. All patients, relatives and controls underwent olfactory function testing. Of these subjects, 23 patients (13 males and 10 females, mean age of 29,3 \pm 8.33 years), 20 unaffected first-degree relatives (13 males and 7 females, mean age of 43,6 \pm 8.6 years) and 26 control subjects (18 males and 8 females, mean age of 38,8 \pm 14.34 years) consented to undergo an additional MRI scan to measure the volume of the OB and the depth of the OS. Of the patients, 10 had an isolated CP, 2 had an isolated CL and 11 had a CLP.

This work was conducted in accordance with the declaration of Helsinki. All participants have given written informed consent. This prospective study was prospectively reviewed and approved by the ethical committee of the University Hospitals Leuven and Catholic University Leuven, Belgium (study ML8636).

\section{Olfactory testing}

Olfactory testing was performed using the validated Sniffin' Sticks identification test (Burghart, Wedel, Germany) (Hummel et al, 1997), with bilateral presentation of the sticks. Odor identification was done by presenting the subject 12 odors. Subjects had to identify the odor out of a list of four possibilities. Based on the score (the higher the score, the better the olfactory capacity), the subject was defined to be normosmic, hyposmic (score <9/12) or 
anosmic (score $<6 / 12$ ), based on normative curves.

MRI

Subjects were examined using a standardized protocol for OB and OS analysis on a 3T magnetic resonance imaging system (Achieva, Philips Healthcare, Best, the Netherlands) with an eight-channel phased-array head coil. The protocol included: (1) 5-mm-thick standard T2-weighted fast spin-echo images covering the whole brain without interslice gap to rule out any organic brain disorder, (2) 2-mm-thick T2-weighted fast spin-echo images in the coronal plane covering the anterior and middle segments of the base of the skull with the following parameters: TR 1,737 ms; TE 90 ms; ETL 13; and slice thickness 2 mm without interslice gap, FOV $170 \mathrm{~mm}$; matrix $512 \times 512$ resulting in a voxel size of $2 \times 0.45 \times 45 \mathrm{~mm}$.

The volume of the $\mathrm{OB}$ and the depth of the OS was calculated on these coronal plane images using a standardized method (Rombaux et al, 2009). The observer browses the coronal T2weighted sections from anterior to posterior and selects the first slice on which the eyeball is no longer visible. This slice location corresponds to the so-called "plane of the posterior tangent through the eyeballs” (PPTE), which cuts the anterior-mid segment of the OB. A straight-line tangent to the surface of the top of the gyrus rectus and to that of the orbital gyrus is drawn using the electronic cursor. The depth of the OS (expressed in $\mathrm{mm}$ ) is measured by drawing a perpendicular line connecting this tangent line to the deepest point of the sulcus.

To perform the OB volumetry, the posterior end of the OB has been determined as the location were two successive slice measurements yield the same result, indicating the beginning of the olfactory tract. The observer performed the analysis twice following this standardized method to identify the intra-rater variability. A second observer performed the measurements in order to calculate the inter-rater variability. The Spearman correlation between both measurements was determined to indicate consistency of the measurements. 
OB volumes were calculated using the in-house developed MevisLab software application 'HEINO' (University Hospitals Leuven, Belgium) which easies the planimetric manual contouring and which automatically calculates the volume after delineation of the OB structure on each slice of the MR image.

\section{Statistical analysis}

Statistical analysis was done using the statistical software package Graphpad Prism 5. Results of olfactory testing were compared using a $\mathrm{Chi}^{2}$ test. OB volumes and OS depths were compared between different groups using an unpaired T-test (all data were normally distributed, confirmed by a Shapiro-Wilk Test for normality). Spearman correlation was calculated to investigate the inter- and intra-rater variability of measurements. All tests were performed at the $5 \%$ significance level.

\section{RESULTS}

\section{Olfactory testing}

There were significantly more patients and relatives with reduced olfactory function than controls ( $p=0,002$ and $p=0,001$, respectively) (Figure 1$)$. One patient was anosmic ( $2 \%)$, 17 were hyposmic (32\%) and 36 were normosmic (66\%). Three unaffected relatives were anosmic (7\%), 12 were hyposmic (27\%) and 29 were normosmic (66\%). None of the control subjects were anosmic, six were hyposmic (17\%) and 29 were normosmic (83\%). The mean Sniffin’ Sticks Scores did not significantly differ between patient $(10,36 \pm 1,9)$, relative $(10,11$ $\pm 2,3)$, and control $(10,68 \pm 1,7)$ groups. There was no significant difference in olfactory capacity between patients with CL, CP and CLP $(p=0,31)$ (Supplementary Figure 1). A reduced olfactory function was more prevalent in obligate carriers than in the other relatives $(p=0,04)$ (Supplementary Figure 2). Unaffected first-degree relatives were significantly older than the 
controls $(\mathrm{p}=0,01)$.

MRI analysis (Table 2)

Significant Spearman correlations $(\mathrm{p}<0.0001)$ were found when comparing the repeated OB volume and OS depth measurements, indicating low intra-rater and inter-rater variabilities. Anosmic patients and relatives were categorized as being hyposmic in this analysis, since this group was too small to include as a separate group in the statistical model.

In hyposmic patients, the OB volumes (left and right) were significantly smaller than in controls (both hyposmic and normosmic) $(\mathrm{p}=0,01$ and $\mathrm{p}=0,003)$ (Figure 2). These effects were not significant in hyposmic relatives compared to controls $(\mathrm{p}=0,27$ and $\mathrm{p}=0,05)$ (Figure 3). The depth of the left OS was significantly smaller in hyposmic patients and relatives ( $p=0,02$ and $\mathrm{p}=0,02$ ) (Figures 4 and 5). The right sulcus however was not significantly smaller in hyposmic patients compared to controls ( $\mathrm{p}=0,26)$, but the right olfactory sulcus was smaller in hyposmic relatives compared to controls $(\mathrm{p}=0,03)$ (Figures 4 and 5). No structural differences that could result in a conductive loss of smell were noticed on the MRI scans of hyposmic subjects.

\section{DISCUSSION}

Although olfactory function of patients with NSCL/P has not been extensively investigated, there is some evidence for a decreased capacity to smell in a significant proportion of these patients. In all previously described studies however, methodological objections can be raised, for instance the use of non-validated methods or the lack of a control group.

In this study, using a validated smell test, the prevalence of hyposmia and anosmia was significantly higher in patients with NSCL/P and their unaffected first-degree relatives than in a control group without family history of orofacial clefting. Structural nasal anomalies may 
play a role in the etiology of smell deficits in patients born with a CL (Drake et al, 1993; Kunkel et al, 1999), for instance due to vestibular stenosis or nasal septum deviation. In our study, patients with structural nasal disorders and nasal obstruction, possibly resulting in a conductive smell deficit, were excluded by taking their history, acoustic rhinometry and rhinomanometry and evaluation of the MRI scans in the subgroup that underwent imaging. Moreover, we tested olfactory function in patients with different cleft types and found reduced olfactory function to be equally prevalent in patients with isolated $\mathrm{CP}$, who are not expected to have structural nasal anomalies. This indicates that structural nasal anomalies are not sufficient to explain the increased prevalence of olfactory deficits in patients with orofacial clefting.

Further and in line with previous research (May et al., 2015; Roosenboom et al., 2015), reduced olfactory function was also more prevalent in unaffected relatives of persons with NSCL/P than in the control group without family history of orofacial clefting. This supports a common basis for the reduced olfactory function and thus identifies a reduced olfactory capacity as an endophenotype of NSCL/P. In obligate carriers, who are by definition carriers of NSCL/P susceptibility factors, the prevalence of reduced olfactory function is higher, in line with the definition of an endophenotype.

In addition, we measured the volume of the OB and the depth of the OS on MR images. The correlation between OB volumes or OS depths and olfactory function is extensively described. Buschhüter et al. described significant correlations between OB volumes and olfactory function, independent of the subjects' age, although OB volumes decrease with age (Buschhüter et al., 2008).

Hyposmic patients with NSCL/P have significantly smaller OB volumes compared to controls. This is in accordance with similar studies in other patient groups, such as patients with congenital anosmia, patients with chronic rhinosinusitis and patients with posttraumatic olfactory loss (Haehner et al, 2008; Levy et al, 2013; Yousem et al, 1996). In hyposmic 
relatives, no significant differences in OB volumes were found as compared to controls. It remains to be investigated if changes in olfactory bulb volume are a primary genetic effect (as in Kallmann syndrome) or a secondary effect of the decreased smell capacity of these patients. There is indeed constant synaptogenesis in the $\mathrm{OB}$, so this structure remains plastic throughout life (Hummel et al, 2015; Levy et al., 2013). This means that there can be an increase in OB volume with improved olfactory function (Hummel et al., 2003).

Only the left OS depth is smaller in hyposmic patients with NSCL/P, which is in accordance to previous studies. Hummel et al. previously described a smaller left OS in hyposmic subjects (controls and patients with chronic rhinosinusitis) and noted that the right OS was significantly deeper than the left OS in controls ('Abolmaali et al, 2002; Hummel et al., 2015)'. Abolmaali et al. also described a smaller left OS depth in patients with congenital anosmia (Abolmaali et al., 2002). This is thus not a specific finding for patients with orofacial clefts. Though differences in brain morphology are described in patients with NSCL/P, they are not lateralized (Nopoulos et al, 2007; Weinberg et al., 2013). It is known that there is a preference for left-sided CL, which might be correlated with the lateralization of the OS depth in hyposmic patients. In this study, the number of patients with a unilateral left-sided CL was not sufficient to confirm this.

In hyposmic relatives however, the OS depths were significantly smaller on both sides compared to controls. Further, previous research showed a smaller upper nasal region in hyposmic unaffected relatives of patients with NSCL/P (Roosenboom et al., 2015). Expansion of the study group (especially the amount of non-affected relatives) is necessary to draw correct conclusions about the nature of the central olfactory changes in patients with NSCL/P and their relatives.

Although not investigated in this study, it would be interesting to correlate OB volumes and OS depths with unilateral olfactory testing so differences between left and right nostrils can be 
ruled out.

One of the limitations of this study is that we did not perform genetic testing to exclude with certainty the rare monogenic causes of clefting that can be associated with smell deficits, for instance Kallmann Syndrome (OMIM: 308700) or CHARGE Syndrome (OMIM: 214800) (Buschhüter et al., 2008; Félix et al, 2006). Another limitation is the use of the Sniffin’ Sticks identification test, instead of the extended test measuring threshold, discrimination and identification (TDI) scores. It has indeed been described that the correlation between the test and re-test results are higher when the complete TDI score is taken into account instead of solely the identification score (Kobal et al., 2000). It is thought that the identification and discrimination tests reflect central olfactory processing, while the threshold component reflects peripheral aspects of olfactory function (Hummel \& Welge-Lüssen, 2006). Therefore, using the complete TDI test could give more information about the nature of the shown smell deficits in patients with NSCL/P and their unaffected relatives. Furthermore, olfactory function is known to decrease with age. Since the unaffected relatives in our study sample were significantly older than the controls (44.9 \pm 8.75 versus $40.5 \pm 13.66$; $\mathrm{p}=0.01)$, an age bias could be suspected. Normative data show that olfaction scores measured by Sniffin' Sticks start to decline after an age of $50^{(27)}$. However, in this study population, all patients, relatives and controls older than 50 years old were normosmic, except for one unaffected relative. This subject (female, 69 years old) was anosmic, with a Sniffin’ Sticks score too low (3/12) to be explained by an age effect alone. In view of these results, we consider a significant age bias unlikely in this dataset. Furthermore, the reduced olfactory function in patients might be due to cleft-related issues early in life, such as scar tissue om the nasal mucosa after surgical repair. Moreover, midfacial hypoplasia, not uncommon in cleft individuals, may cause reduced function of the olfactory region.

Our results support the hypothesis that reduced olfactory function is an endophenotype 
of orofacial clefting, where genes responsible for orofacial clefting may have a pleiotropic effect and are also influencing olfactory functioning. However, it is worth mentioning that there are other theories, although often lacking empirical evidence, which could explain the effect seen in these patients and unaffected relatives. One of them is the evo-devo origin of the nose, as described by Jankowski and colleagues (Jankowski and Marquez, 2016), who states that the formation of the palate is directly related to the formation of the olfactory nose, providing a possible explanation for the effect seen in this study.

In conclusion, patients with NSCL/P and their unaffected relatives have an increased prevalence of smell deficits, associated with changes in the central olfactory structures. This suggests that the underlying susceptibility genes for NSCL/P also influence olfactory structures and function in a subgroup of patients and their relatives. Reduced olfactory function is thus an endophenotype of NSCL/P.

\section{ACKNOWLEDGEMENTS}

G. Hens is supported by a grant from the Research Fund Flanders. P. Claes is supported by Catholic University of Leuven CREA Research Fund. We want to thank the participants of the study for their cooperation and the Multidisciplinary Cleft Team of the University Hospitals Leuven for their support. 


\section{REFERENCES}

Abolmaali, N. D., Hietschold, V., Vogl, T. J., Hüttenbrink, K.-B., \& Hummel, T. (2002). MR evaluation in patients with isolated anosmia since birth or early childhood. AJNR. American Journal of Neuroradiology, 23(1), 157-164.

Buschhüter, D., Smitka, M., Puschmann, S., Gerber, J. C., Witt, M., Abolmaali, N. D., \& Hummel, T. (2008). Correlation between olfactory bulb volume and olfactory function. NeuroImage, 42(2), 498-502. https://doi.org/10.1016/j.neuroimage.2008.05.004

Drake, A. F., Davis, J. U., \& Warren, D. W. (1993). Nasal airway size in cleft and noncleft children. The Laryngoscope, 103(8), 915-917. https://doi.org/10.1288/00005537199308000-00014

Félix, T. M., Hanshaw, B. C., Mueller, R., Bitoun, P., \& Murray, J. C. (2006). CHD7 gene and non-syndromic cleft lip and palate. American Journal of Medical Genetics Part A, 140A(19), 2110-2114. https://doi.org/10.1002/ajmg.a.31308

Gottesman, I. I., \& Gould, T. D. (2003). The Endophenotype Concept in Psychiatry: Etymology and Strategic Intentions. American Journal of Psychiatry, 160(4), 636-645. https://doi.org/10.1176/appi.ajp.160.4.636

Grossmann, N., Brin, I., Aizenbud, D., Sichel, J.-Y., Gross-Isseroff, R., \& Steiner, J. (2005). Nasal airflow and olfactory function after the repair of cleft palate (with and without cleft lip). Oral Surgery, Oral Medicine, Oral Pathology, Oral Radiology, and Endodontology, 100(5), 539-544. https://doi.org/10.1016/j.tripleo.2005.02.074

Haehner, A., Rodewald, A., Gerber, J. C., \& Hummel, T. (2008). Correlation of olfactory function with changes in the volume of the human olfactory bulb. Archives of Otolaryngology--Head \& Neck Surgery, 134(6), 621-624. https://doi.org/10.1001/archotol.134.6.621 
Hummel, T., Damm, M., Vent, J., Schmidt, M., Theissen, P., Larsson, M., \& Klussmann, J.P. (2003). Depth of olfactory sulcus and olfactory function. Brain Research, 975(1), 8589. https://doi.org/10.1016/S0006-8993(03)02589-7

Hummel, T., Sekinger, B., Wolf, S. R., Pauli, E., \& Kobal, G. (1997). “Sniffin” sticks’: olfactory performance assessed by the combined testing of odor identification, odor discrimination and olfactory threshold. Chemical Senses, 22(1), 39-52.

Hummel, T., Urbig, A., Huart, C., Duprez, T., \& Rombaux, P. (2015). Volume of olfactory bulb and depth of olfactory sulcus in 378 consecutive patients with olfactory loss. Journal of Neurology, 262(4), 1046-1051. https://doi.org/10.1007/s00415-015-7691-x Hummel, T., \& Welge-Lüssen, A. (2006). Taste and smell: an update. Basel; New York: Karger.

Jankowsi, R. \& Marquez, S. (2016). Embryology of the nose: the evo-devo concept. World Journal of Otorhinolaryngology, 6(2), 33-40.

Kobal, G., Klimek, L., Wolfensberger, M., Gudziol, H., Temmel, A., Owen, C. M., ... Hummel, T. (2000). Multicenter investigation of 1,036 subjects using a standardized method for the assessment of olfactory function combining tests of odor identification, odor discrimination, and olfactory thresholds. European Archives of Oto-RhinoLaryngology: Official Journal of the European Federation of Oto-Rhino-Laryngological Societies (EUFOS): Affiliated with the German Society for Oto-Rhino-Laryngology Head and Neck Surgery, 257(4), 205-211.

Kunkel, M., Wahlmann, U., \& Wagner, W. (1999). Acoustic airway profiles in unilateral cleft palate patients. The Cleft Palate-Craniofacial Journal: Official Publication of the American Cleft Palate-Craniofacial Association, 36(5), 434-440. https://doi.org/10.1597/1545-1569(1999)036<0434:AAPIUC>2.3.CO;2 Leboyer, M., Bellivier, F., Nosten-Bertrand, M., Jouvent, R., Pauls, D., \& Mallet, J. (1998). 
Psychiatric genetics: search for phenotypes. Trends in Neurosciences, 21(3), 102-105.

Levy, L. M., Degnan, A. J., Sethi, I., \& Henkin, R. I. (2013). Anatomic olfactory structural abnormalities in congenital smell loss: magnetic resonance imaging evaluation of olfactory bulb, groove, sulcal, and hippocampal morphology. Journal of Computer Assisted Tomography, 37(5), 650-657. https://doi.org/10.1097/RCT.0b013e31829bfa3b

Mani, M., Morén, S., Thorvardsson, O., Jakobsson, O., Skoog, V., \& Holmström, M. (2010). Objective Assessment of the Nasal Airway in Unilateral Cleft Lip and Palate-A LongTerm Study. The Cleft Palate-Craniofacial Journal, 47(3), 217-224. https://doi.org/10.1597/09-057.1

Marazita, M. L. (2012). The Evolution of Human Genetic Studies of Cleft Lip and Cleft Palate. Annual Review of Genomics and Human Genetics, 13(1), 263-283. https://doi.org/10.1146/annurev-genom-090711-163729

May, M. A., Sanchez, C. A., Deleyiannis, F. W. B., Marazita, M. L., \& Weinberg, S. M. (2015). Evidence of olfactory deficits as part of the phenotypic spectrum of nonsyndromic orofacial clefting. The Journal of Craniofacial Surgery, 26(1), 84-86. https://doi.org/10.1097/SCS.0000000000001242

Nopoulos, P., Langbehn, D. R., Canady, J., Magnotta, V., \& Richman, L. (2007). Abnormal brain structure in children with isolated clefts of the lip or palate. Archives of Pediatrics \& Adolescent Medicine, 161(8), 753-758. https://doi.org/10.1001/archpedi.161.8.753

Richman, R. A., Sheehe, P. R., McCanty, T., Vespasiano, M., Post, E. M., Guzi, S., \& Wright, H. (1988). Olfactory deficits in boys with cleft palate. Pediatrics, 82(6), 840-4. Retrieved from http://www.ncbi.nlm.nih.gov/pubmed/3186373

Rombaux, P., Grandin, C., \& Duprez, T. (2009). How to measure olfactory bulb volume and olfactory sulcus depth? B-ENT, 5 Suppl 13, 53-60.

Roosenboom, J., Claes, P., Devriendt, K., Dormaar, T., Peeters, H., Saey, I., ... Hens, G. 
(2015). Review: Facial endophenotypes in non-syndromic orofacial clefting. B-ENT.

Roosenboom, J., Saey, I., Peeters, H., Devriendt, K., Claes, P., \& Hens, G. (2015). Facial

Characteristics and Olfactory Dysfunction: Two Endophenotypes Related to

Nonsyndromic Cleft Lip and/or Palate. BioMed Research International, 2015.

Stanier, P. (2004). Genetics of cleft lip and palate: syndromic genes contribute to the incidence of non-syndromic clefts. Human Molecular Genetics, 13(90001), 73R-81. https://doi.org/10.1093/hmg/ddh052

Weinberg, S. M., Parsons, T. E., Fogel, M. R., Walter, C. P., Conrad, A. L., \& Nopoulos, P. (2013). Corpus callosum shape is altered in individuals with nonsyndromic cleft lip and palate. American Journal of Medical Genetics. Part A, 161A(5), 1002-1007.

https://doi.org/10.1002/ajmg.a.35835

Yousem, D. M., Geckle, R. J., Bilker, W., McKeown, D. A., \& Doty, R. L. (1996). MR evaluation of patients with congenital hyposmia or anosmia. AJR. American Journal of Roentgenology, 166(2), 439-443. 


\section{FIGURE LEGENDS}

Figure 1: Olfactory capacity in patients with NSCL/P, their unaffected relatives and controls.

Olfactory testing using the Sniffin' Sticks identification test showed a significantly reduced olfactory function in patients with NSCL/P and their unaffected relatives than in controls ( $p=0,002$ and $p=0,001$, respectively). The red lines indicate the cut-off values for the Sniffin' Sticks (<9/12: hyposmia; <6/12: anosmia).

Figure 2: OB volumes in patients with NSCL/P

In hyposmic patients, the OB volumes (left and right) are significantly smaller than in controls $(\mathrm{p}=0,01$ and $\mathrm{p}=0,003)$.

Figure 3: OB volumes in unaffected relatives of patients with NSCL/P Hyposmic relatives did not show significantly smaller OB volumes, compared to the controls $(p=0,27$ and $p=0,05)$.

Figure 4: OS depths in patients with NSCL/P

The left OS was significantly smaller in hyposmic patients, compared to controls $(p=0,02)$. There was no significant difference in right OS depth.

Figure 5: OS depths in unaffected relatives of patients with NSCL/P

Left and right OS depths were smaller in unaffected relatives compared to controls ( $p=0,02$ and $\mathrm{p}=0,03)$.

Supplementary figure 1: Olfactory capacity and cleft type 
Olfactory capacity in patients with different cleft types was investigated using the Sniffin' Sticks identification test. There was no significant difference in olfactory capacity between patients with CL, CP and CLP ( $\mathrm{p}=0,31)$.

Supplementary figure 2: Olfactory capacity in obligate carriers

Obligate carriers tend to have a reduced olfactory function than relatives without a family member with orofacial clefting, apart from the index patient $(p=0,04)$. 


\section{TABLES}

\begin{tabular}{|l|l|l|l|l|}
\hline \multicolumn{1}{|c|}{ Group } & \multicolumn{1}{|c|}{ Anosmia } & \multicolumn{1}{|c|}{ Hyposmia } & Normosmia & Total \\
\hline Patients & 1 & 17 & 36 & 54 \\
\hline Relatives (non- & 3 & 12 & 29 & 44 \\
obligate carriers) & & & & 12 \\
\hline Obligate carriers & 2 & 3 & 7 & 35 \\
\hline Controls & 0 & 6 & 29 & \\
\hline
\end{tabular}

Table 1: Number of subjects in the olfaction study

The numbers of tested subjects that are anosmic, hyposmic or normosmic are displayed. 


\begin{tabular}{|c|c|c|c|c|c|}
\hline Group & $\begin{array}{l}\text { Olfactory } \\
\text { function }\end{array}$ & $\begin{array}{l}\text { OB left } \\
\left(\mathrm{mm}^{3}\right)\end{array}$ & $\begin{array}{c}\text { OB right } \\
\left(\mathrm{mm}^{3}\right)\end{array}$ & $\begin{array}{l}\text { OS left } \\
\text { (mm) }\end{array}$ & $\begin{array}{c}\text { OS right } \\
\text { (mm) }\end{array}$ \\
\hline \multirow[t]{2}{*}{$\begin{array}{l}\text { Patients } \\
(n=23)\end{array}$} & Hyposmia (n=8) & $\begin{array}{l}51,83 \pm \\
12,85 *\end{array}$ & $\begin{array}{l}52,27 \pm \\
13,36 * *\end{array}$ & $\begin{array}{l}5,75 \pm 0,97 \\
*\end{array}$ & $6,51 \pm 1,13$ \\
\hline & $\begin{array}{l}\text { Normosmia } \\
(n=15)\end{array}$ & $\begin{array}{l}65,3 \pm \\
12,92\end{array}$ & $\begin{array}{l}66,53 \pm \\
16,58\end{array}$ & $7,07 \pm 1,16$ & $7,23 \pm 1,21$ \\
\hline \multirow[t]{2}{*}{$\begin{array}{l}\text { Relatives } \\
(n=20)\end{array}$} & Hyposmia $(n=6)$ & $\begin{array}{l}55,7 \pm \\
30,21\end{array}$ & $\begin{array}{l}55,45 \pm \\
26,62\end{array}$ & $\begin{array}{l}4,97 \pm 2,66 \\
*\end{array}$ & $5,38 \pm 2,6 *$ \\
\hline & $\begin{array}{l}\text { Normosmia } \\
(n=14)\end{array}$ & $\begin{array}{l}70,61 \pm \\
15,58\end{array}$ & $\begin{array}{l}79,42 \pm \\
16,94\end{array}$ & $5,88 \pm 1,63$ & $6,8 \pm 1,67$ \\
\hline $\begin{array}{l}\text { Controls } \\
(n=26)\end{array}$ & $\begin{array}{l}\text { Normosmia } \\
(n=22)\end{array}$ & $68,8 \pm 15,4$ & $\begin{array}{l}73,57 \pm \\
16,74\end{array}$ & $6,77 \pm 1,11$ & $7,08 \pm 1,21$ \\
\hline
\end{tabular}

Table 2: OB volumes and OS depths

Mean OB volumes and OS depths ( \pm standard deviation) of hyposmic and normosmic patients and relatives, compared to normosmic controls, are shown. The olfactory function of the subjects was determined using the Sniffin' Sticks identification test. The number of subjects in each group are described.

$*: \mathrm{p}<0,05$

$* *: \mathrm{p}<0,01$ 